\title{
Variance-minimization scheme for optimizing Jastrow factors
}

\author{
N. D. Drummond and R. J. Needs \\ TCM Group, Cavendish Laboratory, University of Cambridge, Madingley Road, Cambridge CB3 OHE, United Kingdom
}

(Received 9 May 2005; revised manuscript received 23 June 2005; published 18 August 2005)

\begin{abstract}
We describe a scheme for optimizing many-electron trial wave functions by minimizing the unreweighted variance of the energy using stochastic integration and correlated-sampling techniques. The scheme is restricted to parameters that are linear in the exponent of a Jastrow correlation factor, which are the most important parameters in the wave functions we use. The scheme is highly efficient and allows us to investigate the parameter space more closely than has been possible before. We search for multiple minima of the variance in the parameter space and compare the wave functions obtained using reweighted and unreweighted variance minimization.
\end{abstract}

DOI: 10.1103/PhysRevB.72.085124

PACS number(s): 71.15.Dx, 02.70.Ss, 31.25.-v

\section{INTRODUCTION}

Accurate many-body wave functions are essential to the variational and diffusion quantum Monte Carlo (VMC and DMC) methods, as the wave function controls both the statistical efficiency and the accuracy of these techniques. ${ }^{1}$ Optimizing many-body wave functions is perhaps the most important technical issue facing practitioners of these quantum Monte Carlo (QMC) techniques today, and it consumes large quantities of human and computing resources.

Wave-function optimization schemes have usually involved minimizing either the variational energy or its variance. Although it is generally believed that wave functions corresponding to the minimum energy have more desirable properties, variance minimization has been very widely used because it has proved easier to design robust minimization techniques for this purpose. ${ }^{2,3}$ The scheme introduced in this paper involves minimizing the unreweighted variance. We describe an alternative method for evaluating this quantity, which greatly accelerates the optimization of parameters that occur in a linear fashion in the exponent of a Jastrow factor. The optimization step does not involve a sum over electron configurations, which means that we can use very large numbers of configurations. The unreweighted variance is in fact a quartic function of the linear parameters in the Jastrow factor, and the minima of multidimensional quartic functions can be located very rapidly. The efficiency of our scheme has enabled us to explore the minimization procedure and the parameter space in detail, and to investigate the possible existence of multiple minima.

The distinction between the reweighted or true variance and the unreweighted variance is explained in Sec. II. In Sec. III we describe our accelerated scheme for calculating the unreweighted variance. In Sec. IV we use our method to study the unreweighted variance in parameter space. The minima of the reweighted and unreweighted variance need not coincide, and in Sec. V we investigate which minimum corresponds to the lower energy. We discuss the sampling of configuration space and the flexibility of the trial wave function in Secs. VI and VII. A smooth scheme for removing "outlying" configurations is studied in Sec. VIII. In Secs. IX and X we compare the efficiency of the "standard" and accelerated variance-minimization methods, both in theory and practice. Finally, we draw our conclusions in Sec. XI.

Hartree atomic units (a.u.) are used throughout, in which the Dirac constant, the magnitude of the electronic charge, the electronic mass, and $4 \pi$ times the permittivity of free space are unity: $\hbar=|e|=m_{e}=4 \pi \epsilon_{0}=1$. All of our QMC calculations were carried out using the CASINO package. ${ }^{4}$

\section{ENERGY AND ITS VARIANCE}

Consider a real trial wave function $\Psi(\mathbf{R})$, where $\mathbf{R}$ is a point in the electron configuration space. In VMC the energy is written as

$$
E=\frac{\int \Psi(\mathbf{R})^{2} E_{L}(\mathbf{R}) d \mathbf{R}}{\int \Psi(\mathbf{R})^{2} d \mathbf{R}},
$$

where the local energy $E_{L}$ is

$$
E_{L}(\mathbf{R})=\Psi(\mathbf{R})^{-1} \hat{H}(\mathbf{R}) \Psi(\mathbf{R}),
$$

and $\hat{H}$ is the Hamiltonian. The variance of the energy is

$$
\sigma^{2}=\frac{\int \Psi(\mathbf{R})^{2}\left[E_{L}(\mathbf{R})-E\right]^{2} d \mathbf{R}}{\int \Psi(\mathbf{R})^{2} d \mathbf{R}} .
$$

We write the trial wave function as $\Psi^{\{\alpha\}}(\mathbf{R})$, to denote that it depends on a set of free parameters $\{\alpha\}$. Consider a set of $N_{C}$ configurations $\{\mathbf{R}\}$ distributed according to $\left[\Psi^{\left\{\alpha_{0}\right\}}(\mathbf{R})\right]^{2}$ for some fixed parameter set $\left\{\alpha_{0}\right\}$. The variance $\sigma^{2}$ is then estimated for any given parameter set $\{\alpha\}$ using a correlatedsampling procedure, which gives rise to the reweighted variance

$$
\sigma_{w}^{2}=\frac{T_{\left\{\alpha_{0}\right\}}^{\alpha \alpha\}}}{\left(T_{\left\{\alpha_{0}\right\}}^{\alpha \alpha\}}\right)^{2}-\sum_{\mathbf{R}}\left(W_{\left\{\alpha_{0}\right\}}^{\{\alpha\}}(\mathbf{R})\right)^{2}} \sum_{\mathbf{R}}\left(E_{L}^{\{\alpha\}}(\mathbf{R})-\bar{E}_{w}\right)^{2} W_{\left\{\alpha_{0}\right\}}^{\alpha \alpha\}}(\mathbf{R}),
$$


where the reweighted energy is

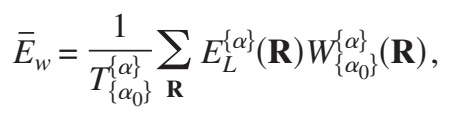

which is an estimate of $E$, and the total weight is

$$
T_{\left\{\alpha_{0}\right\}}^{\alpha \alpha\}}=\sum_{\mathbf{R}} W_{\left\{\alpha_{0}\right\}}^{\alpha \alpha\}}(\mathbf{R}),
$$

and the weights $W$ are

$$
W_{\left\{\alpha_{0}\right\}}^{\{\alpha\}}(\mathbf{R})=\left(\frac{\Psi^{\{\alpha\}}(\mathbf{R})}{\Psi^{\left\{\alpha_{0}\right\}}(\mathbf{R})}\right)^{2} .
$$

Throughout this paper, we confine our attention to the optimization of parameters in the Jastrow factor. The nodal surface of the trial wave function is independent of such parameters, so the weights cannot diverge when the parameters change.

The unreweighted variance as a function of parameter set $\{\alpha\}$ is defined to be

$$
\sigma_{u}^{2}=\frac{1}{N_{C}-1} \sum_{\mathbf{R}}\left(E_{L}^{\{\alpha\}}(\mathbf{R})-\bar{E}_{u}\right)^{2},
$$

where the unreweighted energy is

$$
\bar{E}_{u}=\frac{1}{N_{C}} \sum_{\mathbf{R}} E_{L}^{\{\alpha\}}(\mathbf{R}) .
$$

The reweighted and unreweighted variances are identical when the same set of configurations is used and $\{\alpha\}=\left\{\alpha_{0}\right\}$. However, for any given $\left\{\alpha_{0}\right\}$ they are different functions of $\{\alpha\}$, and there is no reason to expect that their minima coincide with each other, or that either minimum should coincide with that of the (reweighted) energy.

Both $\sigma_{w}^{2}$ and $\sigma_{u}^{2}$ are non-negative, but are zero when $\Psi^{\{\alpha\}}$ is an eigenstate of $\hat{H}$. The reweighted and unreweighted variances are therefore reasonable cost functions for wavefunction optimizations. The reweighted energy is also a reasonable cost function. However, the problem with the reweighted energy and variance is that the weights $W$ may vary rapidly as the parameters change, especially for large systems, which leads to instabilities in optimization procedures. ${ }^{3}$ It is shown in Appendix A that the wave function used to generate the configuration set corresponds to a stationary point of $\bar{E}_{u}$ (for perfect sampling). In what follows we will mainly be interested in optimizing linear parameters in the Jastrow factor, and in this case the wave function used to generate the configuration set corresponds to the global maximum of the unreweighted energy, as proved in Appendix B. The unreweighted energy is clearly not a suitable cost function. From these considerations we conclude that the cost function with the most suitable mathematical properties for the stable optimization of wave functions within the correlated-sampling approach is the unreweighted variance.

The usual variance-minimization procedure is to generate a set of electron configurations $\{\mathbf{R}\}$ distributed according to $\left[\Psi^{\left\{\alpha_{0}\right\}}(\mathbf{R})\right]^{2}$ using VMC, and then to minimize the reweighted or unreweighted energy variance over this set.
Since the variance landscape depends on the distribution of configurations, several cycles of configuration generation and optimization are normally carried out, with the optimized wave function from the previous cycle being used in each VMC configuration-generation phase. We usually iterate several times and choose the wave function that gives the lowest variational energy. In the limit of perfect sampling, the reweighted variance is equal to the actual variance, and is therefore independent of the configuration distribution, so that the optimized parameters would not change over successive cycles of reweighted variance minimization. This is not the case for unreweighted variance minimization; nevertheless, by carrying out a number of cycles, a "self-consistent" parameter set may be obtained.

\section{ACCELERATED EVALUATION OF THE UNREWEIGHTED VARIANCE}

\section{A. Slater-Jastrow wave function}

Let $\Psi$ be a Slater-Jastrow wave function for a many-body system

$$
\Psi(\mathbf{R})=\exp [J(\mathbf{R})] S(\mathbf{R}),
$$

where $\exp [J]$ is the Jastrow factor, which contains free parameters to be determined by an optimization method, and $S$ is the Slater wave function, which may be an expansion in several determinants of single-particle orbitals. is,

Suppose that $J$ contains linear parameters $\alpha_{1}, \ldots, \alpha_{P}$, that

$$
J(\mathbf{R})=\sum_{i=1}^{P} f_{i}(\mathbf{R}) \alpha_{i}+J_{0}(\mathbf{R}),
$$

where $f_{1}, \ldots, f_{P}$ and $J_{0}$ are known functions of $\mathbf{R}$, which depend upon the particular form of Jastrow factor used and do not contain any free parameters. We use the form of Jastrow factor described in detail in Ref. 5, which contains linear parameters. However, some of the terms have a finite extent in space and the associated cut-off lengths must appear nonlinearly in the Jastrow factor. These cut-off lengths can be set on physical grounds or optimized using small numbers of parameters and configurations and the standard variance-minimization procedure, but their values cannot be obtained using the accelerated scheme described here.

\section{B. Derivation of the quartic polynomial}

The local energy for the Slater-Jastrow wave function of Eq. (10) is

$$
\begin{aligned}
E_{L}(\mathbf{R})= & -\frac{1}{2} \sum_{i=1}^{P} \sum_{j=1}^{P} g_{i j}^{(2)}(\mathbf{R}) \alpha_{i} \alpha_{j} \\
& -\frac{1}{2} \sum_{i=1}^{P} g_{i}^{(1)}(\mathbf{R}) \alpha_{i}-\frac{1}{2} g^{(0)}(\mathbf{R})+V(\mathbf{R}),
\end{aligned}
$$

where $V$ is the potential energy and

$$
g_{i j}^{(2)}(\mathbf{R})=\nabla f_{i} \cdot \nabla f_{j},
$$




$$
\begin{gathered}
g_{i}^{(1)}(\mathbf{R})=2 \nabla f_{i} \cdot \nabla J_{0}+\nabla^{2} f_{i}+2 \frac{\nabla S}{S} \cdot \nabla f_{i}, \\
g^{(0)}(\mathbf{R})=\left|\nabla J_{0}\right|^{2}+\nabla^{2} J_{0}+2 \frac{\nabla S}{S} \cdot \nabla J_{0}+\frac{\nabla^{2} S}{S},
\end{gathered}
$$

and we note that $g_{i j}^{(2)}=g_{j i}^{(2)}$. The square of the local energy is given by

$$
\begin{aligned}
E_{L}^{2}(\mathbf{R})= & \sum_{i=1}^{P} \sum_{j=1}^{P} \sum_{k=1}^{P} \sum_{l=1}^{P} G_{i j k l}^{(4)}(\mathbf{R}) \alpha_{i} \alpha_{j} \alpha_{k} \alpha_{l} \\
& +\sum_{i=1}^{P} \sum_{j=1}^{P} \sum_{k=1}^{P} G_{i j k}^{(3)}(\mathbf{R}) \alpha_{i} \alpha_{j} \alpha_{k} \\
& +\sum_{i=1}^{P} \sum_{j=1}^{P} G_{i j}^{(2)}(\mathbf{R}) \alpha_{i} \alpha_{j} \\
& +\sum_{i=1}^{P} G_{i}^{(1)}(\mathbf{R}) \alpha_{i}+G^{(0)}(\mathbf{R}),
\end{aligned}
$$

where

$$
\begin{gathered}
G_{i j k l}^{(4)}(\mathbf{R})=\frac{g_{i j}^{(2)}(\mathbf{R}) g_{k l}^{(2)}(\mathbf{R})}{4}, \\
G_{i j k}^{(3)}(\mathbf{R})=\frac{g_{i j}^{(2)}(\mathbf{R}) g_{k}^{(1)}(\mathbf{R})}{2}, \\
G_{i j}^{(2)}(\mathbf{R})=\frac{g_{i}^{(1)}(\mathbf{R}) g_{j}^{(1)}(\mathbf{R})}{4}-g_{i j}^{(2)}(\mathbf{R})\left(V(\mathbf{R})-\frac{g^{(0)}(\mathbf{R})}{2}\right), \\
G_{i}^{(1)}(\mathbf{R})=-g_{i}^{(1)}(\mathbf{R})\left(V(\mathbf{R})-\frac{g^{(0)}(\mathbf{R})}{2}\right), \\
G^{(0)}(\mathbf{R})=\left(V(\mathbf{R})-\frac{g^{(0)}(\mathbf{R})}{2}\right)^{2} .
\end{gathered}
$$

(Note that $\quad G_{i j k l}^{(4)}=G_{j i k l}^{(4)}=G_{i j l k}^{(4)}=G_{k l i j}^{(4)}, \quad G_{i j k}^{(3)}=G_{j i k}^{(3)}, \quad$ and $G_{i j}^{(2)}=G_{j i}^{(2)}$.)

Suppose the VMC method is used to generate a set of $N_{C}$ points in configuration space, $\{\mathbf{R}\}$, which are distributed according to the square of an approximate trial wave function. For any quantity $A(\mathbf{R})$, let

$$
\bar{A}=\frac{1}{N_{C}} \sum_{\mathbf{R}} A(\mathbf{R})
$$

be the average of $A(\mathbf{R})$ over the set of $N_{C}$ configurations. The unreweighted variance may be written as

$$
\begin{aligned}
\sigma_{u}^{2}= & \frac{N_{C}}{N_{C}-1}\left(\bar{E}_{L}^{2}-\bar{E}_{L}^{2}\right) \equiv \frac{N_{C}}{N_{C}-1}\left(\sum_{i=1}^{P} \sum_{j=1}^{P} \sum_{k=1}^{P} \sum_{l=1}^{P} K_{i j k l}^{(4)} \alpha_{i} \alpha_{j} \alpha_{k} \alpha_{l}\right. \\
& +\sum_{i=1}^{P} \sum_{j=1}^{P} \sum_{k=1}^{P} K_{i j k}^{(3)} \alpha_{i} \alpha_{j} \alpha_{k}+\sum_{i=1}^{P} \sum_{j=1}^{P} K_{i j}^{(2)} \alpha_{i} \alpha_{j} \\
& \left.+\sum_{i=1}^{P} K_{i}^{(1)} \alpha_{i}+K^{(0)}\right)
\end{aligned}
$$

where

$$
\begin{gathered}
K_{i j k}^{(3)}=\bar{G}_{i j k}^{(3)}-\frac{\bar{g}_{i j}^{(2)} \bar{g}_{k}^{(1)}}{2}, \\
K_{i j}^{(2)}=\bar{G}_{i j}^{(2)}-\frac{\bar{g}_{i}^{(1)} \bar{g}_{j}^{(1)}}{4}+\bar{g}_{i j}^{(2)}\left(\bar{V}-\frac{\bar{g}^{(0)}}{2}\right), \\
K_{i}^{(1)}=\bar{G}_{i}^{(1)}+\bar{g}_{i}^{(1)}\left(\bar{V}-\frac{\bar{g}^{(0)}}{2}\right), \\
K^{(0)}=\bar{G}^{(0)}-\left(\bar{V}-\frac{\bar{g}^{(0)}}{2}\right)^{2} .
\end{gathered}
$$

$\left(\right.$ Note that $K_{i j k l}^{(4)}=K_{j i k l}^{(4)}=K_{i j l k}^{(4)}=K_{k l i j}^{(4)}, K_{i j k}^{(3)}=K_{j i k}^{(3)}$, and $K_{i j}^{(2)}=K_{j i}^{(2)}$.) The unreweighted variance is quartic in the set of free parameters. Once the values of $K^{(n)}$ have been computed, there is no need to perform any further summations over the set of configurations during the optimization of the parameters.

Throughout this paper the potential energy is assumed to be a local operator, so the local potential energy is independent of the wave-function parameters. When the varianceminimization algorithm is applied to systems containing pseudoatoms, the change in the local potential energy due to the nonlocal part of the pseudopotential is neglected. Not only does this greatly improve the speed of the varianceminimization process, but it also appears to improve the stability of the algorithm.

\section{Evaluating the least-squares function during an optimization}

\section{Accumulating $\overline{\boldsymbol{G}}$ and $\bar{g}$}

The values of $\bar{g}, \bar{G}$, and $\bar{V}$ are accumulated during a VMC simulation by keeping a running total of the values of $g(\mathbf{R}), G(\mathbf{R})$, and $V(\mathbf{R})$ encountered at each step of the random walk; there is no need to store data for each configuration. The accumulated elements of $G$ are stored in a onedimensional array and, furthermore, the symmetries of $G$ are exploited in order to minimize the length of this vector. The numbers of $G^{(4)}, G^{(3)}, G^{(2)}, G^{(1)}$, and $G^{(0)}$ elements to be calculated and stored are

$$
\begin{gathered}
N_{G}^{(4)}=\frac{\left(\frac{P(P+1)}{2}\right)\left(\frac{P(P+1)}{2}+1\right)}{2}, \\
N_{G}^{(3)}=\frac{P^{2}(P+1)}{2},
\end{gathered}
$$




$$
\begin{gathered}
N_{G}^{(2)}=\frac{P(P+1)}{2}, \\
N_{G}^{(1)}=P, \\
N_{G}^{(0)}=1,
\end{gathered}
$$

respectively. $G_{i j k l}^{(4)}$ is symmetric with respect to $i$ and $j$, and is also symmetric with respect to $k$ and $l$. In order to label the independent elements of $G^{(4)}$, one can replace $(i, j)$ by a single index $I$ that takes $P(P+1) / 2$ different values. Likewise, $(k, l)$ can be replaced by a single index $J$ that takes $P(P+1) / 2$ different values. $G_{I J}^{(4)}$ is still symmetric with respect to $I$ and $J$; hence $(I, J)$ can be replaced by a single index $K$ which takes $N_{G}^{(4)}$ different values, where $N_{G}^{(4)}$ is given in Eq. (29). This is the method by which the elements of $G^{(4)}$ are indexed in practice. Counting and indexing the elements of $G^{(3)}, G^{(2)}$, and $G^{(1)}$ are relatively straightforward. The total number of $G$ elements grows as $O\left(P^{4}\right)$. Storing these coefficients represents the memory bottleneck for the accelerated optimization procedure. With $P=30$ parameters (a typical number), $122791 G$ elements must be stored. With $P=100$ parameters (a large number), 13263926 elements must be stored. Alternatively, the number of elements to be stored could be reduced by using the same strategy as that suggested in Sec. III C 2 for evaluating the unreweighted variance. This would not affect the number of elements that have to be evaluated, however, and it may slow down the VMC calculation even further. The saving in memory would typically be a factor between 2.5 and 3, which is insignificant, given the $O\left(P^{4}\right)$ scaling of the method.

\section{Evaluating the least-squares function}

Before the start of the optimization, the coefficient of each different product of parameters is computed and the coefficients are stored in a one-dimensional array. This allows the unreweighted variance to be evaluated extremely rapidly. The set of possible products of four of the parameters is $\left\{\alpha_{i} \alpha_{j} \alpha_{k} \alpha_{l}: i \leqslant j \leqslant k \leqslant l\right\}$, and similarly for the products of three and two parameters. So the unreweighted variance can be written as

$$
\begin{aligned}
\sigma_{u}^{2}= & \frac{N_{C}}{N_{C}-1}\left(\Gamma^{(0)}+\sum_{i=1}^{P} \alpha_{i}\left\{\Gamma_{i}^{(1)}+\sum_{j=i}^{P} \alpha_{j}\left[\Gamma_{i j}^{(2)}+\sum_{k=j}^{P} \alpha_{k}\right.\right.\right. \\
& \left.\left.\left.\times\left(\Gamma_{i j k}^{(3)}+\sum_{l=k}^{P} \alpha_{l} \Gamma_{i j k l}^{(4)}\right)\right]\right\}\right),
\end{aligned}
$$

where the $\Gamma^{(n)}$ are defined in terms of $K^{(n)}$ (see below) and are stored as one-dimensional arrays. The number of elements of $\Gamma^{(n)}$ is given by the number of distinct products of $n$ parameters, which can be shown to be

$$
N_{T}^{(n)}=\left(\begin{array}{c}
P+n-1 \\
n
\end{array}\right),
$$

while the total number of elements of the $\Gamma$ arrays is

$$
N_{T}=\left(\begin{array}{c}
P+4 \\
4
\end{array}\right),
$$

which increases as $O\left(P^{4}\right)$. For $P=30$ parameters, the number of terms that must be summed over to obtain the unreweighted variance is 46376 , while for $P=100$ parameters, the number of terms is 4598126.

For each $\{i, j, k, l\}$ with $i \leqslant j \leqslant k \leqslant l, \Gamma_{i j k l}^{(4)}$ is equal to the sum of $K_{i j k l}^{(4)}$ over all distinct permutations of $\{i, j, k, l\}$. $\Gamma^{(3)}, \Gamma^{(2)}, \Gamma^{(1)}$, and $\Gamma^{(0)}$ are constructed in a similar fashion.

\section{Derivatives of the least-squares function}

Derivatives of the unreweighted variance are given by

$$
\begin{aligned}
\frac{\partial \sigma_{u}^{2}}{\partial \alpha_{n}}= & \frac{N_{C}}{N_{C}-1}\left(\sum_{i=1}^{P} \sum_{j=1}^{P} \sum_{k=1}^{P} M_{i j k}^{(3)}(n) \alpha_{i} \alpha_{j} \alpha_{k}\right. \\
& \left.+\sum_{i=1}^{P} \sum_{j=1}^{P} M_{i j}^{(2)}(n) \alpha_{i} \alpha_{j}+\sum_{i=1}^{P} M_{i}^{(1)}(n) \alpha_{i}+M^{(0)}(n)\right),
\end{aligned}
$$

where

$$
\begin{gathered}
M_{i j k}^{(3)}(n)=K_{n i j k}^{(4)}+K_{i n j k}^{(4)}+K_{i j n k}^{(4)}+K_{i j k n}^{(4)}, \\
M_{i j}^{(2)}(n)=K_{n i j}^{(3)}+K_{i n j}^{(3)}+K_{i j n}^{(3)} \\
M_{i}^{(1)}(n)=K_{n i}^{(2)}+K_{i n}^{(2)} \\
M^{(0)}(n)=K_{n}^{(1)} .
\end{gathered}
$$

In practice, derivatives are evaluated as

$$
\begin{aligned}
\frac{\partial \sigma_{u}^{2}}{\partial \alpha_{n}}= & \frac{N_{C}}{N_{C}-1}\left\{\Lambda^{(0)}(n)+\sum_{i=1}^{P} \alpha_{i}\left[\Lambda_{i}^{(1)}(n)\right.\right. \\
& \left.\left.+\sum_{j=i}^{P} \alpha_{j}\left(\Lambda_{i j}^{(2)}(n)+\sum_{k=j}^{P} \alpha_{k} \Lambda_{i j k}^{(3)}(n)\right)\right]\right\},
\end{aligned}
$$

where the $\Lambda(n)$ are defined in terms of the $M(n)$ in an analogous fashion to the definition of $\Gamma$ in terms of $K$ in Sec. III $C 2$. The total number of elements of $\Lambda$ is

$$
N_{T}^{\prime}=P\left(\begin{array}{c}
P+3 \\
3
\end{array}\right),
$$

which grows as $O\left(P^{4}\right)$. The $\Lambda$ arrays used to evaluate the gradient of the unreweighted variance may be somewhat larger than the $G$ arrays.

\section{Minimizing the variance}

Ideally, one would like to use an optimization method that enables one to find the global minimum of the variance with respect to the wave-function parameters. Unfortunately, existing variance-minimization algorithms generally use numerical optimization methods which, if started close to a particular local minimum, will always converge to that minimum. However, in the case of the quartic unreweighted vari- 
ance in the space of linear Jastrow parameters, it is relatively easy to carry out an extensive search for the global minimum.

Standard methods for minimizing a function of many variables include the method of steepest descents, the conjugate-gradients method, and the Broyden-FletcherGoldfarb-Shanno (BFGS) method. ${ }^{6}$ Of these three methods, we have found the BFGS algorithm to converge most rapidly for a wide variety of test systems.

Along any given line in the space of linear Jastrow parameters the unreweighted variance is a quartic polynomial of a single variable. The method by which the variance along a line can be reexpressed as a quartic polynomial is given in Appendix C. A quartic polynomial of a single variable has at most two minima on the real axis. The gradient of a quartic function is a cubic, whose three roots can be obtained analytically; ${ }^{6}$ hence it is straightforward to locate the global minimum of the unreweighted variance along the line.

In order to search the parameter space for the global minimum of the variance with respect to the linear Jastrow parameters, we first perform a BFGS minimization. Starting from this minimum we choose directions at random and use the analytical line-minimization technique to search for a second minimum, lower than the first. If a second minimum is found then BFGS is used to converge to the new minimum, and the process is repeated.

\section{NATURE OF THE UNREWEIGHTED VARIANCE}

\section{A. Linear Jastrow parameters}

We used the method described in Sec. III D to search for minima when optimizing the linear Jastrow parameters in the $\mathrm{SiH}_{4}$ molecule, the all-electron neon atom, a 16-atom cell of diamond-structure pseudosilicon subject to periodic boundary conditions, and an electron-hole gas. However, even sampling up to $10^{7}$ random directions, multiple minima were only found when the configurations were distributed according to an extremely poor wave function and few configurations were used.

Plots of the unreweighted variance against the value of one of the linear parameters are shown for an all-electron neon atom in Fig. 1. It can be seen that the unreweighted variance converges to a limit as the number of configurations is increased. There is only one minimum in every case.

Plots of the unreweighted variance of an all-electron neon atom against the value of a parameter for an extremely poor distribution of configurations are shown in Fig. 2. When few configurations are used $\left(N_{C}=40\right)$, it is possible to find two minima of the variance along lines in parameter space, proving that nonglobal minima can exist. However, it is also found that increasing the number of configurations tends to prevent the occurrence of two minima along lines in parameter space.

\section{B. Nonlinear Jastrow parameters}

Plots of the unreweighted variance of the $\mathrm{SiH}_{4}$ molecule against a nonlinear Jastrow parameter-the cut-off length for the electron-electron correlation term ${ }^{5}$-are shown in Figs. 3

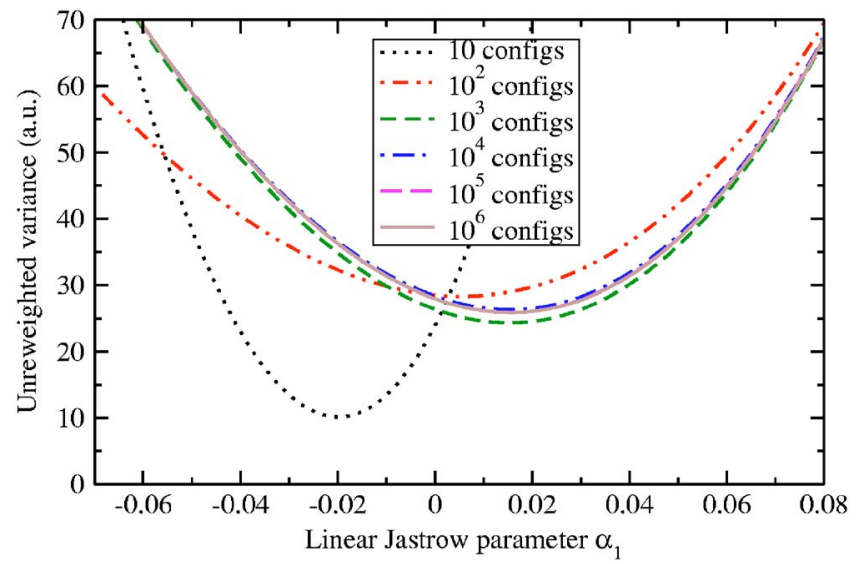

FIG. 1. (Color online) The unreweighted variance $\sigma_{u}^{2}$ for an all-electron neon atom plotted against one of the linear Jastrow parameters. All of the other linear parameters are set to zero. Different numbers of configurations were used to calculate the quartic coefficients of the unreweighted variance. In each case the configurations were distributed according to the square of the Hartree-Fock wave function. The Jastrow factor contained a total of 24 linear parameters. The curves for $10^{5}$ and $10^{6}$ configurations are indistinguishable in the figure.

and 4. The behavior of the unreweighted variance is far worse when the cut-off length is varied than when a linear parameter is varied: The variance has multiple minima along lines in parameter space and there is some noise in the variance, especially for poor samplings of configuration space. It can be seen in Fig. 4 that the optimized cut-off lengths obtained using $10^{2}$ or $10^{3}$ configurations are considerably

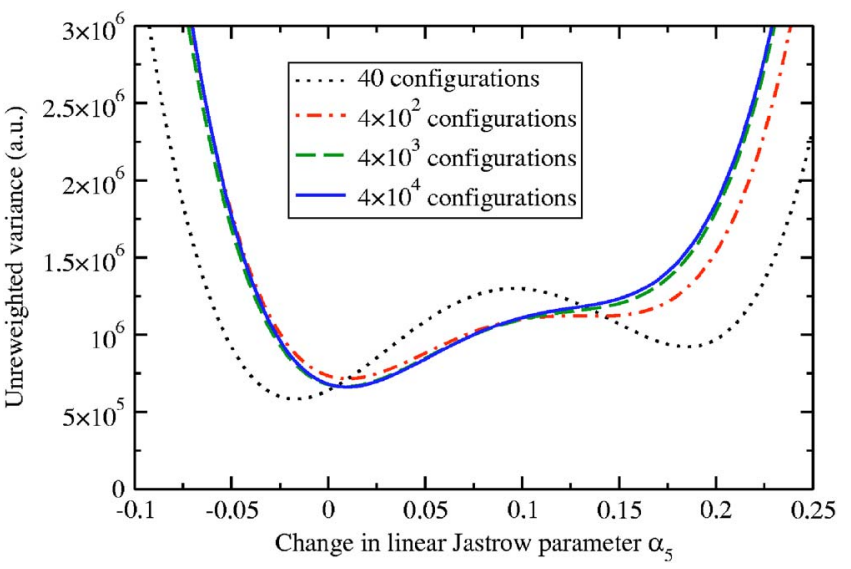

FIG. 2. (Color online) The unreweighted variance $\sigma_{u}^{2}$ for an all-electron neon atom plotted against the change in one of the linear Jastrow parameters. All the parameters in the Jastrow factor are set to large, random values. Different numbers of VMCgenerated configurations were used to calculate the quartic coefficients of the unreweighted variance. In each case the configurations were distributed according to the square of a Slater-Jastrow wave function, where the Jastrow factor contained the random parameters, so that the resulting distribution was very unlike the groundstate distribution. The Jastrow factor contained a total of 72 linear parameters. The Slater wave function contained Hartree-Fock orbitals. 


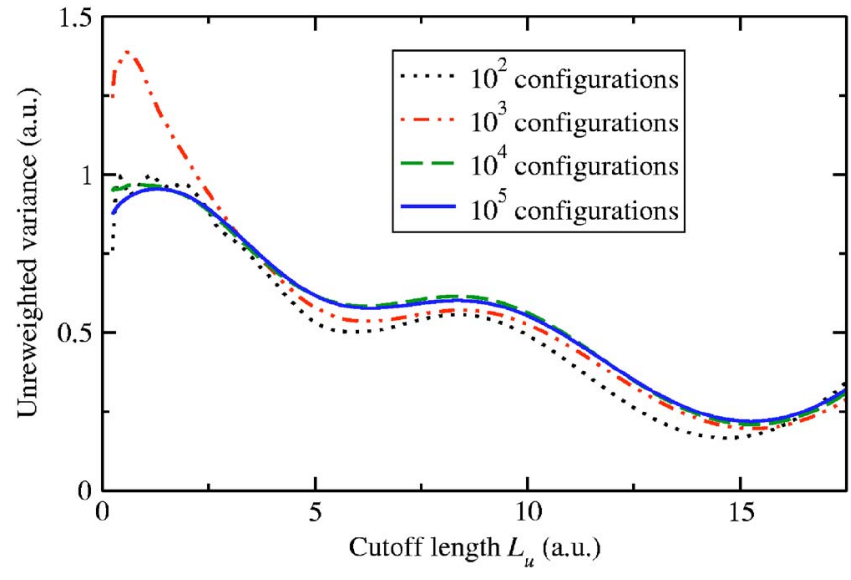

FIG. 3. (Color online) The unreweighted variance $\sigma_{u}^{2}$ for a $\mathrm{SiH}_{4}$ molecule [with a Hartree-Fock silicon pseudopotential (Ref. 7)] plotted against the cutoff length for the electron-electron terms in the Jastrow factor $L_{u}$. The Jastrow factor is such that the local energy is continuous when an electron-electron separation passes through the cut-off length (Ref. 5). Different numbers of VMCgenerated configurations were used to calculate the unreweighted variance. All of the linear Jastrow parameters are set to zero. In each case the configurations were distributed according to the square of the Hartree-Fock wave function. The Jastrow factor contained a total of 56 linear parameters, plus three cut-off lengths.

shorter than the cut-off lengths obtained using $10^{4}$ or $10^{5}$ configurations. In the former case the cut-off lengths are trapped in the nonglobal minimum that can be seen in Fig. 3, while the deeper minimum is reached in the latter case. The Jastrow factor used to produce Figs. 3 and 4 is such that the local energy is continuous when an electron-electron separation passes through the cut-off length. If a Jastrow factor that gives rise to a discontinuous local energy at the cut-off length were to be used, the variance would be an extremely noisy function of the cut-off length, especially for thin samplings of configuration space. Optimization of the cut-off lengths for such Jastrow factors has been found to be very difficult. ${ }^{5}$ The existence of multiple minima when cut-off lengths are optimized suggests that it may be worthwhile

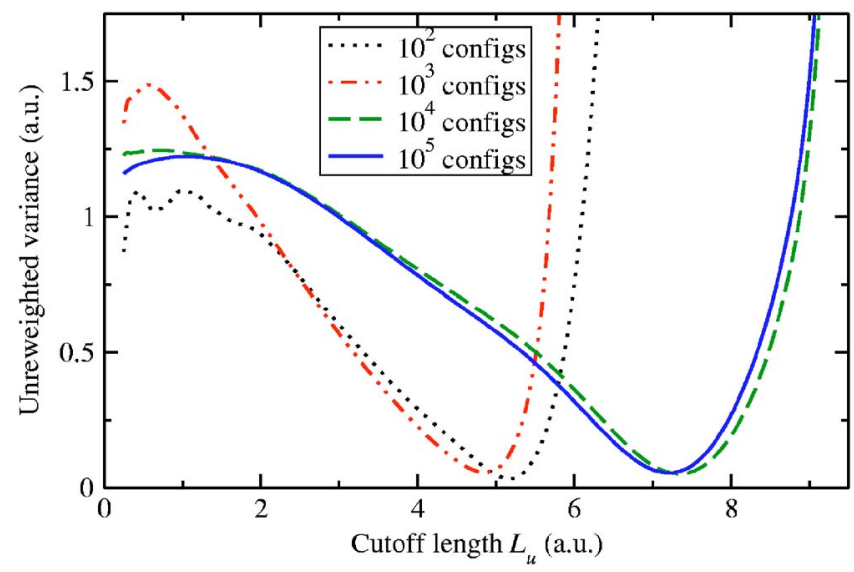

FIG. 4. (Color online) The same as Fig. 3, except that all of the parameters in the Jastrow factor (including the cut-off lengths) have been optimized.

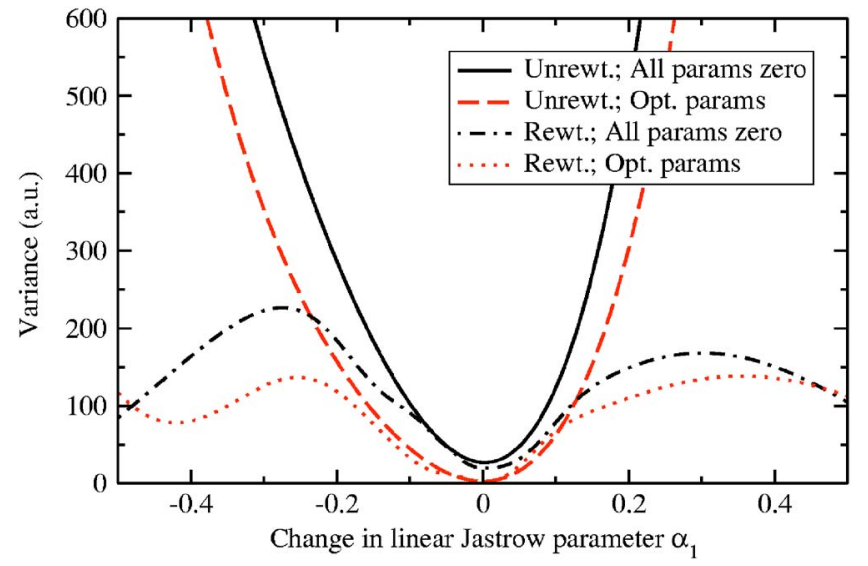

FIG. 5. (Color online) The reweighted and unreweighted variance for an all-electron neon atom plotted against the change in the value of a linear Jastrow parameter $\alpha_{1}$. Plots are shown for the case in which all the parameters are set to zero and the case in which all the parameters have been optimized. The set of 100 configurations used to calculate the variance were distributed according to the square of the Hartree-Fock wave function. The Jastrow factor contained a total of 27 linear parameters.

performing variance-minimization calculations using several different initial cut-off lengths.

\section{MINIMA OF THE VARIANCE AND THE ENERGY}

\section{A. Reweighted and unreweighted variance}

Plots of the reweighted and unreweighted variances for an all-electron neon atom against one of the linear Jastrow parameters are shown in Figs. 5 and 6 for small and large numbers of configurations. The reweighted and unreweighted variances have their minima in different places, with their values at the minima being different from one another. The variance is a smooth function of the linear Jastrow parameter in each case, but there are multiple minima of the reweighted variance along lines in parameter space, demonstrating that nonglobal minima can exist. Furthermore, the minima of the reweighted variance are not as sharply

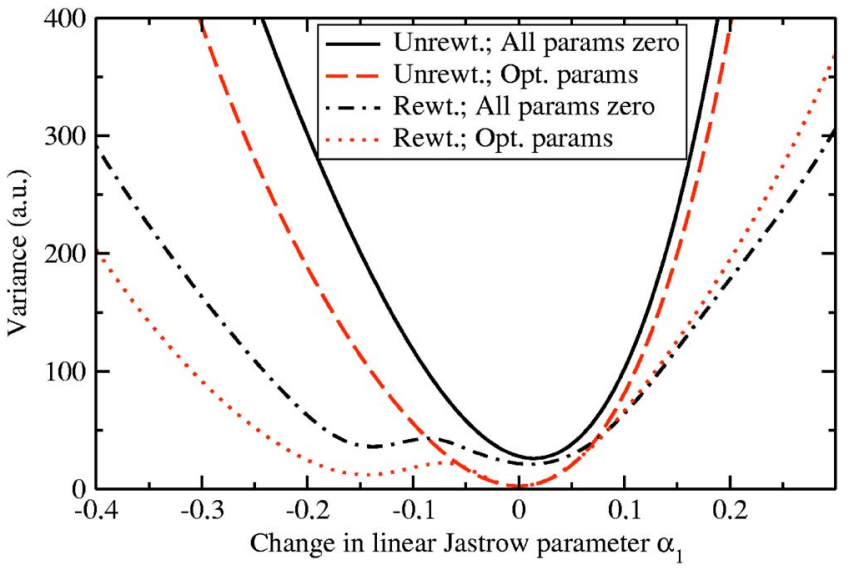

FIG. 6. (Color online) The same as Fig. 5 except that $10^{4}$ configurations were used to calculate the variance. 
TABLE I. Results of reweighted and unreweighted variance-minimization calculations for an all-electron neon atom. $P$ is the number of linear parameters in the Jastrow factor and $N_{C}$ is the number of configurations used to perform the optimization. (Long VMC runs were used to obtain the energies and variances shown in the table.) Only linear Jastrow parameters were optimized. The VMC energy and variance for cycle 1 are estimates of the Hartree-Fock energy and variance, and are the same for each $P$ and $N_{C}$.

\begin{tabular}{|c|c|c|c|c|c|c|}
\hline \multirow[b]{2}{*}{$P$} & \multirow[b]{2}{*}{$N_{C}$} & \multirow[b]{2}{*}{ Cycle } & \multicolumn{2}{|c|}{ VMC energy (a.u.) } & \multicolumn{2}{|c|}{ Variance (a.u.) } \\
\hline & & & Unrew. & Rew. & Unrew. & Rew. \\
\hline 1 & 500 & 1 & $-128.5469(5)$ & $-128.5469(5)$ & 29.3(1) & 29.3(1) \\
\hline 1 & 500 & 2 & $-128.5424(3)$ & $-128.5858(3)$ & $6.3786(7)$ & $6.220(2)$ \\
\hline 1 & 500 & 3 & $-128.6178(3)$ & $-128.6129(3)$ & $6.310(1)$ & $6.207(1)$ \\
\hline 1 & 500 & 4 & $-128.6267(3)$ & $-86.508(3)$ & $6.1564(7)$ & $1501.1(2)$ \\
\hline 1 & $5 \times 10^{5}$ & 2 & $-128.6170(3)$ & $-128.6248(3)$ & $6.116(2)$ & $6.0848(9)$ \\
\hline 1 & $5 \times 10^{5}$ & 3 & $-128.6226(3)$ & $-128.6261(3)$ & $6.096(1)$ & $6.0844(8)$ \\
\hline 1 & $5 \times 10^{5}$ & 4 & $-128.6201(3)$ & $-128.6260(3)$ & $6.103(1)$ & $6.0834(9)$ \\
\hline 72 & 500 & 2 & $-128.87184(9)$ & $-128.86664(9)$ & $1.4178(7)$ & $1.3733(8)$ \\
\hline 72 & 500 & 3 & $-128.88040(9)$ & $-50.00(9)$ & $1.358(1)$ & $420.7(86)$ \\
\hline 72 & 500 & 4 & $-128.8749(1)$ & $258.83(1)$ & $1.421(2)$ & $13599.1(4816)$ \\
\hline 72 & $5 \times 10^{5}$ & 2 & $-128.89760(7)$ & $-128.89622(7)$ & $1.136(1)$ & $1.1330(9)$ \\
\hline 72 & $5 \times 10^{5}$ & 3 & $-128.89742(7)$ & $-128.89677(7)$ & $1.1343(7)$ & $1.132(1)$ \\
\hline 72 & $5 \times 10^{5}$ & 4 & $-128.89752(7)$ & $-128.89655(7)$ & $1.137(2)$ & $1.1326(9)$ \\
\hline
\end{tabular}

defined as those of the unreweighted variance. Minimization of the unreweighted variance is therefore more likely to be rapid and stable.

The outcomes of actual reweighted and unreweighted variance-minimization calculations are shown in Table I. For a relatively sparse sampling of configuration space, reweighted variance minimization is pathologically unstable, while unreweighted variance minimization is perfectly wellbehaved. For a dense sampling of configuration space the two methods give very similar results, and there is no evidence that the reweighted variance-minimization algorithm performs any better than the unreweighted algorithm, or vice versa.

\section{B. Coincidence of the minima of the energy and the variance}

As is clearly demonstrated in Fig. 7, the self-consistent minimum of the unreweighted variance does not necessarily coincide with the minimum of the VMC energy. On the other hand, for a high-quality Jastrow factor, ${ }^{8}$ the minima of the unreweighted variance and energy are generally in close agreement, as is shown in Fig. 8. We have no evidence, for all-electron atoms at least, that any significant advantage could be obtained by optimizing linear Jastrow parameters in a good Jastrow factor using an energy-minimization method. It can also be seen in Fig. 8 that the reweighted energy follows the actual VMC energy data closely (the statistical error in the reweighted energy at the optimal wave function is 0.001 a.u.). This implies that, provided enough configurations are used, the wave function could be optimized by reweighted energy minimization.

A plot of the VMC energy variance against the change in a linear Jastrow parameter from its optimal value in an allelectron neon atom is shown in Fig. 9. As one would expect, the reweighted variance matches the actual variance, unlike the unreweighted variance; however, there is no significant difference between the minima of the variance and the unreweighted variance (and hence the energy).

We have also studied the question of the coincidence of the minima of the energy, the variance, and the selfconsistent unreweighted variance using a variety of model systems, for which the integrals could be performed exactly. The models consisted of one-dimensional potential wells and various trial wave functions with a single variable parameter. We studied several examples for a single particle and an example for two identical, interacting fermions. These examples showed that the global minima of the energy, the variance, and the self-consistent unreweighted variance can

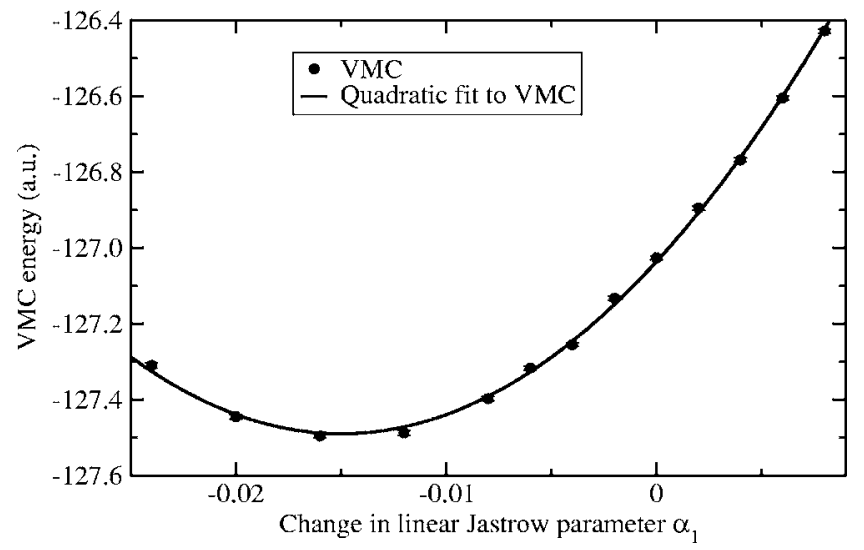

FIG. 7. The VMC energy of an all-electron neon atom against the change in the value of a linear Jastrow parameter $\alpha_{1}$ from the value determined by self-consistent unreweighted variance minimization. The Jastrow factor was chosen to be poor, with no electronnucleus or electron-electron-nucleus terms, and the same electronelectron terms were used for both parallel and antiparallel spins. There is only one optimizable parameter: $\alpha_{1}$. 


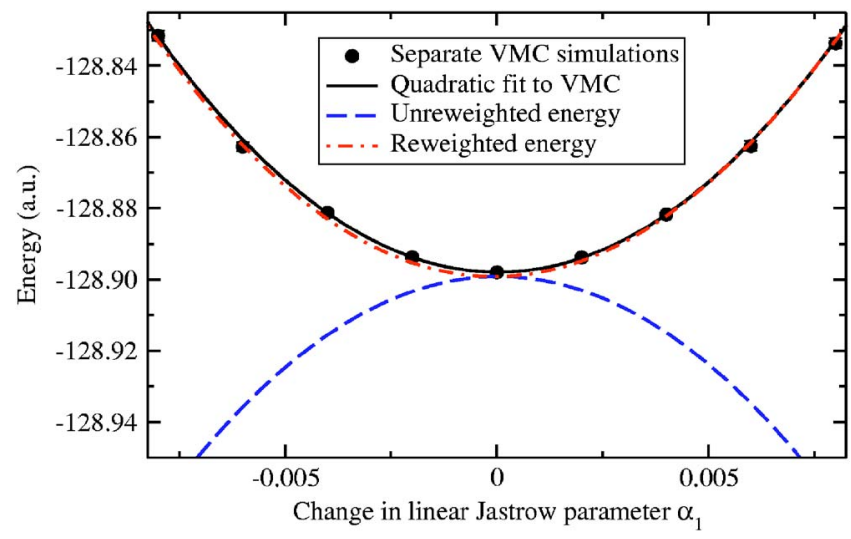

FIG. 8. (Color online) VMC energy of an all-electron neon atom against the change in the value of a linear Jastrow parameter $\alpha_{1}$ from the value determined by self-consistent unreweighted variance minimization. Specifically, the Jastrow factor was the best allelectron neon Jastrow factor described in Ref. 5. The reweighted and unreweighted energies calculated using $8 \times 10^{5}$ configurations distributed according to the square of the optimized wave function are also plotted. The statistical error bars in the VMC data are smaller than the symbols.

be different. In all cases studied the parameters optimized by self-consistent unreweighted variance minimization gave lower energies than the parameters optimized by reweighted or "true" variance minimization. Furthermore, in many cases, the parameters from the self-consistent unreweighted variance minimum coincided exactly with the energyminimized parameters, suggesting that some underlying principle was at work. One such example is given in Appendix D.

\section{SAMPLING OF CONFIGURATION SPACE}

\section{A. Number of configurations}

Suppose we have a poor trial wave function corresponding to some particular parameter set $\left\{\alpha_{0}\right\}$. Consider the surface in configuration space $E_{L}^{\left\{\alpha_{0}\right\}}(\mathbf{R})=c$, where $c$ is a con-

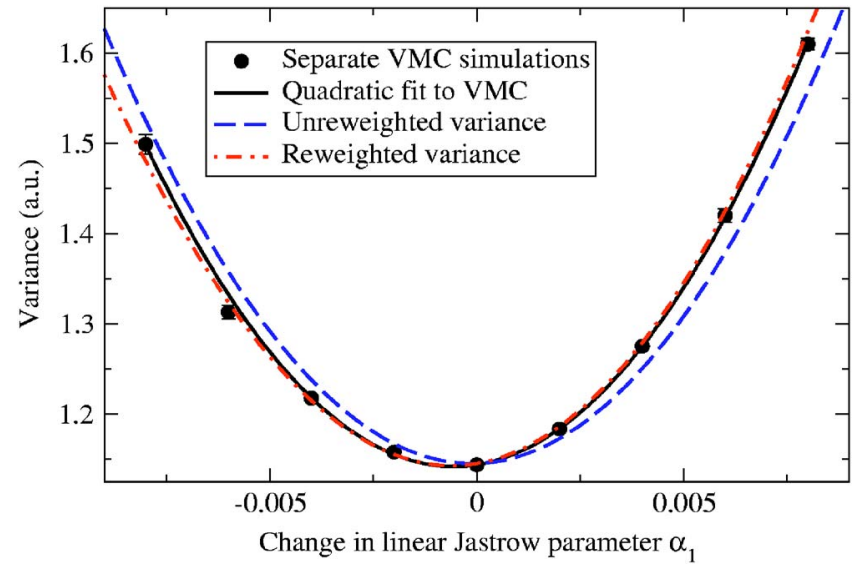

FIG. 9. (Color online) Same as Fig. 8 except that the VMC variance is plotted against the change in the parameter value.

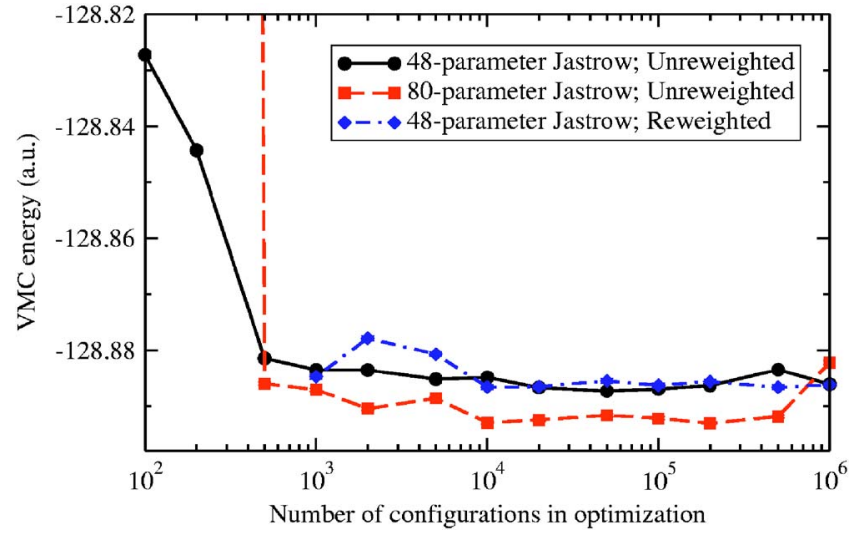

FIG. 10. (Color online) VMC energy of an all-electron neon atom against the number of configurations used to optimize the linear Jastrow parameters in an unreweighted variance minimization. Eight optimization cycles were performed for each number of configurations in order to ensure that self-consistency was achieved. The Slater wave function contained Hartree-Fock orbitals. The error bars in the VMC data are smaller than the symbols.

stant. Let us choose any number $N_{C}$ of configurations to lie on this surface. If this configuration set is used to perform variance minimization then the variance (reweighted or unreweighted) takes its global minimum of zero when the parameter set is $\left\{\alpha_{0}\right\}$. More generally, it is always possible in principle to obtain a poor wave function by locating the global minimum of the variance calculated using a finite sampling of configuration space. Clearly, problems of this sort are more likely to occur if either a poor sampling of configuration space or an over-flexible wave function is used. The effect of changing the sampling of configuration space is studied in this section, while the effect of increasing the flexibility of the Jastrow factor is examined in Sec. VII.

The VMC energy for a neon-atom Slater-Jastrow wave function is plotted against the number of configurations used to optimize the Jastrow factor in Fig. 10. It can be seen that the wave-function quality improves very rapidly, then saturates at between $5 \times 10^{2}$ and $10^{4}$ configurations, for both small and large numbers of parameters. For very small numbers of configurations, the optimizations give pathological results, especially when the more flexible Jastrow factor is used.

Results obtained using reweighted variance minimization are also shown in Fig. 10. The reweighted varianceminimization process was pathologically unstable for fewer than about $10^{3}$ configurations. For larger numbers of configurations the energies obtained are in good agreement with the results of unreweighted variance minimization.

\section{B. Distribution of configurations}

The unreweighted variance for an all-electron neon atom is plotted against a linear Jastrow parameter for three different configuration distributions in Fig. 11. The configurations were distributed according to (i) the square of the HartreeFock wave function, as is usually the case in the first cycle of a variance-minimization calculation; (ii) the square of an op- 


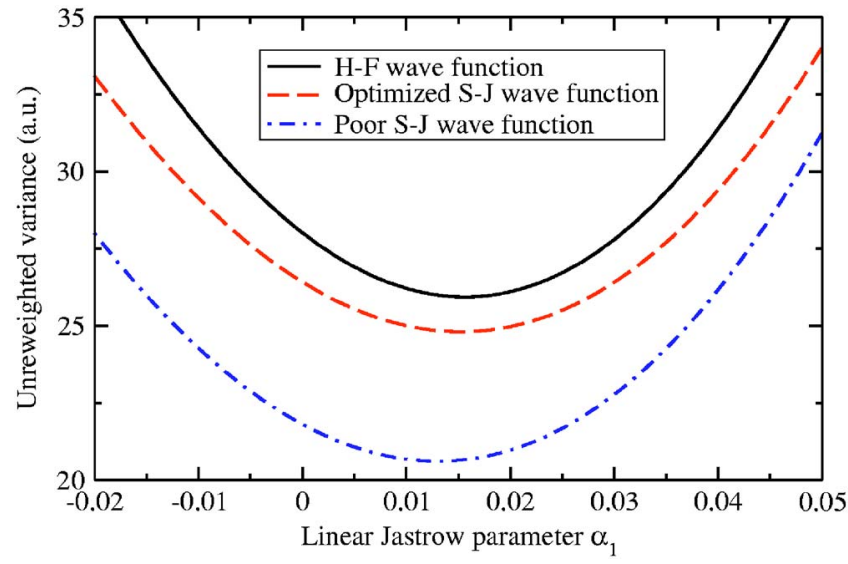

FIG. 11. (Color online) Unreweighted variance for an allelectron neon atom plotted against the value of the linear Jastrow parameter $\alpha_{1}$ for three different configuration distributions: the square of the Hartree-Fock wave function, the square of an optimized Slater-Jastrow wave function, and the square of a poor Slater-Jastrow wave function. $10^{6}$ configurations were used to calculate the unreweighted variance. The Jastrow factor contained a total of 36 linear parameters. The Slater wave function contained Hartree-Fock orbitals.

timized Slater-Jastrow wave function, as is usually the case in the second and subsequent cycles; and (iii) the square of a Slater-Jastrow wave function in which the Jastrow factor was chosen to be poor. Although the variance looks different in each case, the positions of the minima coincide almost exactly for the Slater and optimized Slater-Jastrow distributions. Even for the poor wave function, the minimum of the variance is reasonably close to the more accurately determined optimum. This is consistent with our observation that, in general, the only significant improvement to the quality of a Jastrow factor occurs in the first cycle of a series of unreweighted variance-minimization calculations: Starting from the Hartree-Fock wave function, the self-consistent solution is usually reached in the first cycle.

\section{FLEXIBILITY OF THE JASTROW FACTOR}

The VMC energy of neon is plotted against the number of linear parameters used in the Jastrow factor in Fig. 12. The results illustrate the futility of attempting to optimize too many parameters. The quality of the optimized wave function depends on the number of configurations used to perform the optimization, especially when the number of parameters in the wave function is either very small or very large. However, there would only appear to be an advantage to be gained by using more than $10^{4}$ configurations when a very large number of parameters are to be optimized.

It should be reemphasized that the problems which occur when large numbers of parameters are optimized are caused by mismatches between the minima of the unreweighted variance and the energy due to the finite sampling of configuration space, and not by the introduction of local minima into the variance landscape.

\section{LIMITING OF CONFIGURATION WEIGHTS}

It has been suggested that variance-minimization calculations are disproportionately affected by "outlying" configu-

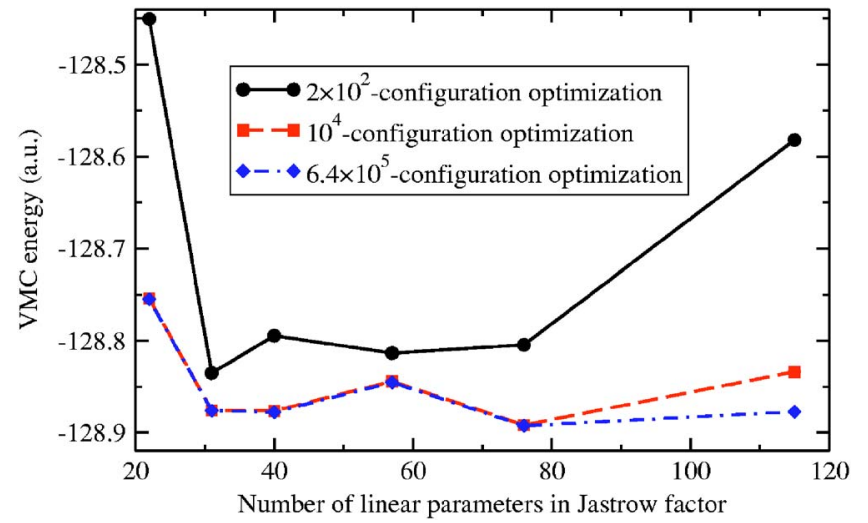

FIG. 12. (Color online) VMC energy of an all-electron neon atom against the number of parameters in the Jastrow factor. Different numbers of configurations were used to carry out the unreweighted variance-minimization calculations. Six optimization cycles were performed in order to guarantee self-consistency. Very long VMC simulations were carried out using the optimized Jastrow factors in order to obtain the energies plotted in the graph. The VMC error bars are smaller than the symbols. The Slater wave function contained Hartree-Fock orbitals.

rations, whose energies deviate substantially from the mean energy. ${ }^{3}$ In particular, the local energy diverges in the vicinity of the nodal surface of the trial wave function, so configurations in this region are especially problematic. Such configurations are relatively rare when the nodes are fixed, as is the case when only Jastrow parameters are optimized, but the problem can be far more serious when parameters that affect the nodal surface are optimized using a fixed sampling of configuration space.

We have studied a smooth scheme for removing outlying configurations from the optimization process. Let us define the configuration "effective weight" to be

$$
W^{\prime}(\mathbf{R})=\frac{1}{2}\left[1-\tanh \left(\frac{\left(E_{L}^{\{\alpha\}}(\mathbf{R})-\bar{E}_{u}\right)^{2}-A^{2} \sigma_{u}^{2}}{B^{2} \sigma_{u}^{2}}\right)\right],
$$

where $\bar{E}_{u}$ and $\sigma_{u}^{2}$ are the unreweighted energy and variance of the set of configurations. $W^{\prime}(\mathbf{R}) \approx 1$ for configurations such that $E_{L}^{\{\alpha\}}(\mathbf{R}) \approx \bar{E}_{u}$, but $W^{\prime}(\mathbf{R}) \rightarrow 0$ for configurations whose local energies are far from the mean. The parameter $A$ is the number of standard deviations of the energy beyond which configurations are excluded, while $B$ is the width of the region in which the effective weights fall off to zero (in terms of standard deviations of the energy). We typically chose $A$ to lie between 2 and 3 and $B$ to lie between $1 / 2$ and 1. The effective weights $W^{\prime}$ are used in place of the weights $W$ in Eq. (4), and the reweighted variance $\sigma_{w}^{2}$ is minimized.

We have found that this weight-limiting scheme is capable of improving the stability of Jastrow-factor optimization when very small numbers of configurations are used. However, the energies of the resulting wave functions are not generally as good as the energies obtained using the same forms of wave function optimized with an adequate number of configurations. For large numbers of configurations, the limiting scheme has very little effect on the optimization of 
Jastrow factors. We conclude that the weight-limiting scheme is not of much practical benefit when a Jastrow factor is to be optimized; however the scheme has been found to be very useful when parameters that affect the nodal surface are optimized. ${ }^{9}$

Other limiting schemes have been devised to improve the stability of the variance-minimization algorithms. For example, it is possible to combine the reweighted and unreweighted variance-minimization algorithms by limiting the values that the weights $W$ can take. ${ }^{10}$ Alternatively, the local energies themselves can be limited. ${ }^{3}$ The latter approach has been found to be problematic, as it can result in spurious minima in the variance corresponding to parameter sets for which a large number of local energies are limited.

\section{SCALING OF THE VARIANCE-MINIMIZATION METHODS}

\section{A. CPU time required for the optimization phase}

Let $N$ be the total number of electrons in a system, $P$ be the number of Jastrow parameters to be optimized, and $N_{C}$ be the number of configurations used to calculate the variance. Although the Jastrow factor of Ref. 5 is considered in this work, the conclusions reached should be valid for most other forms of Jastrow factor in current use.

The computational effort required to evaluate the unreweighted variance using the accelerated method is independent of $N$ and $N_{C}$, but scales as $O\left(P^{4}\right)$. The time taken to compute the gradient of the variance is also $O\left(P^{4}\right)$. It may be assumed that the number of optimization steps required is independent of $P, N$, and $N_{C}$. The $O\left(P^{4}\right)$ scaling of the memory requirements of the accelerated method limits the number of parameters that can be optimized in a single calculation to between 100 and 200, depending on the available memory.

The time taken to recompute the Jastrow factor and its derivatives after all of the parameters have changed is generally $O(N)$ for electron-nucleus and electron-electronnucleus terms and $O\left(N^{2}\right)$ for electron-electron terms. ${ }^{5}$ The CPU time required to evaluate the variance (reweighted or unreweighted) using the standard procedure therefore increases as $O\left(N^{2}\right)$. The time taken to calculate the Jastrow factor is, in general, $O(P)$, and hence the time taken to calculate the variance using the standard method is also $O(P)$. Furthermore, each minimization step requires the gradient of the variance with respect to the parameters, which has $P$ components. The time taken to perform each iteration is therefore $O\left(P^{2}\right)$. The CPU time for the standard method clearly scales as $O\left(N_{C}\right)$.

Putting this together, the CPU time for the optimization phase scales as $O\left(P^{4}\right)$ for the accelerated method and $O\left(N^{2} P^{2} N_{C}\right)$ for the standard method. It should be noted that the time required by the optimization phase in the accelerated scheme is completely negligible in comparison with the time required by the VMC coefficient-gathering phase, whereas the CPU time required by the optimization phase in the standard method is usually rather greater than the CPU time required by the VMC phase.
TABLE II. Timing results for ten cycles of a $6 \times 10^{4}$-configuration unreweighted variance minimization of a 38 linear-parameter Jastrow factor for an all-electron $\mathrm{H}_{2} \mathrm{O}$ molecule. The system contains a total of ten electrons. The Slater wave function contained Hartree-Fock orbitals. The runs were carried out on a $1.7 \mathrm{GHz}$ Pentium processor in a Sony Vaio laptop.

\begin{tabular}{lcc}
\hline \hline Method & Stage & CPU time (s) \\
\hline \multirow{3}{*}{ Standard } & VMC & 5669.43 \\
& Opt. & 58740.65 \\
& Total & 64410.08 \\
Accel. & VMC & 14378.90 \\
& Opt. & 39.69 \\
\hline \hline
\end{tabular}

\section{B. CPU time required for the gathering of the quartic coefficients in the accelerated scheme}

In the standard variance-minimization method, the CPU time required to generate the set of configurations used to compute the variance does not differ appreciably from the time taken to perform an ordinary VMC simulation. For the accelerated optimization method, however, the time taken to compute the quartic expansion coefficients can be a significant fraction of the total CPU time.

The gathering of the quartic coefficients can be divided into two stages: (i) the evaluation of the Jastrow "basis functions" $f_{i}(\mathbf{R})$ for each configuration $\mathbf{R}$ [see Eq. (11)], and (ii) the calculation of the corresponding contributions to the $\bar{g}$ and $\bar{G}$ arrays. Stage (ii) scales as $O\left(P^{4}\right)$, but is independent of system size. By contrast, stage (i) scales as $O(P)$, because there are $P$ basis functions, but the scaling with system size is the same as that of evaluating the Jastrow factor: roughly $O\left(N^{2}\right)$.

The CPU time for an ordinary VMC calculation is generally determined by the time taken to evaluate the orbitals in the Slater wave function. The computational effort required to carry out a fixed number of configuration moves grows as $O\left(N^{2}\right)$ if extended orbitals represented in a localized basis are used. The use of localized orbitals can improve this scaling to $O(N) .{ }^{11}$ In principle the time taken for stage (i) of the coefficient gathering will take up an increasingly large fraction of the CPU time, but in practice the prefactor is so small that the time required is negligible even for the largest systems that we have studied. The time taken for stage (ii) can be the largest contribution to the CPU time for VMC simulations of small molecules, but the effort required is independent of system size, and so, overall, the coefficient-gathering phase of the accelerated scheme is more efficient for large systems than small systems.

\section{$X$. EFFICIENCY OF THE ACCELERATED OPTIMIZATION METHOD}

Timing results for the optimization of the linear Jastrow parameters for an $\mathrm{H}_{2} \mathrm{O}$ molecule (10 electrons) and a $\mathrm{C}_{26} \mathrm{H}_{32}$ molecule (136 electrons) are shown in Tables II and III, re- 
TABLE III. Timing results for four cycles of a $1.6 \times 10^{4}$-configuration unreweighted variance minimization of a 12-linear-parameter Jastrow factor for a $\mathrm{C}_{26} \mathrm{H}_{32}$ molecule with Troullier-Martins carbon and hydrogen pseudopotentials. The system contains a total of 136 electrons. The Slater wave function contained DFT-PBE orbitals. The runs were carried out on a cluster of eight $2.1 \mathrm{GHz}$ Opteron processors.

\begin{tabular}{|c|c|c|}
\hline Method & Stage & time $(\mathrm{s})$ \\
\hline & VMC & 6526.77 \\
\hline \multirow[t]{3}{*}{ Standard } & Opt. & 9323.53 \\
\hline & Total & 15850.30 \\
\hline & VMC & 6828.47 \\
\hline \multirow[t]{2}{*}{ Accel. } & Opt. & 28.14 \\
\hline & Total & 6856.61 \\
\hline
\end{tabular}

spectively. The calculations are fairly typical in terms of the number of parameters and number of configurations. In both cases the use of the accelerated optimization scheme essentially eliminates the cost of the optimization phase. In the standard method the cost of the optimization phase exceeds that of the VMC configuration-generation phase by an order of magnitude for $\mathrm{H}_{2} \mathrm{O}$ and by a less significant proportion for $\mathrm{C}_{26} \mathrm{H}_{32}$. The cost of the VMC phase in the accelerated scheme is increased substantially for $\mathrm{H}_{2} \mathrm{O}$ although, overall, it is still much faster to use the accelerated scheme. For $\mathrm{C}_{26} \mathrm{H}_{32}$ the increase in the CPU time for configuration generation is negligible. Overall, the accelerated optimization scheme is 4.5 times faster for $\mathrm{H}_{2} \mathrm{O}$ and 2.3 times faster for $\mathrm{C}_{26} \mathrm{H}_{32}$.

The actual time taken to compute the variance in the accelerated scheme is minute: On a $2.7 \mathrm{GHz}$ Pentium 4 processor, it takes an average of $83.6 \mu$ s to compute the variance with 25 parameters, while it takes $13.98 \mathrm{~ms}$ to compute the variance with 100 parameters.

\section{CONCLUSIONS}

We have introduced a scheme for evaluating the unreweighted variance of the VMC energy which greatly accelerates the optimization of parameters that occur in a linear fashion in the exponent of a Jastrow factor. This scheme is very efficient because it uses the property that the unreweighted variance is a quartic function of such parameters. We studied a wide range of systems and found that the unreweighted variance almost invariably has a single minimum in the space of the linear parameters. The only exceptions to this that we could find occurred when the configuration space was very poorly sampled, with the configurations being drawn from a distribution completely unlike the square of an eigenfunction of the Hamiltonian. For other wave-function parameters, however, the unreweighted variance often has more than one minimum.

It is easy to use very large numbers of configurations to perform optimizations using our accelerated scheme. We have investigated the effect of varying the number of configurations on the wave-function quality, and we have found that there is, in general, no significant benefit to be obtained from using more than about $10^{4}$ configurations when optimizing linear Jastrow parameters.

We have considered various wave-function optimization schemes using correlated-sampling approaches for minimizing the energy and the variance of the energy. Reweighted energy and variance minimization using correlated sampling suffer from numerical instabilities due to fluctuations in the values of the weights, which are severe for large systems. The unreweighted energy always has a stationary point at the wave function used to generate the configuration set, and for parameters which occur linearly in the Jastrow factor this stationary point is the global maximum in the energy. The unreweighted energy is therefore not a suitable cost function for wave-function optimization. The minima of the variance, the unreweighted variance (iterated to self-consistency), and the energy are generally distinct. In various model systems that we have studied, the self-consistent minimum in the unreweighted variance always gave lower energies than the minimum in the reweighted variance.

\section{ACKNOWLEDGMENTS}

Financial support was provided by the Engineering and Physical Sciences Research Council (EPSRC), UK. Computing resources have been provided by the CambridgeCranfield High Performance Computing Facility.

\section{APPENDIX A: STATIONARY POINT OF THE UNREWEIGHTED ENERGY}

Suppose an extremely large number of configurations are distributed according to $\Psi_{0}^{2}(\mathbf{R})$. Then the unreweighted energy is given by

$$
E_{u}=\frac{\int \Psi_{0}^{2} \Psi^{-1} \hat{H} \Psi d \mathbf{R}}{\int \Psi_{0}^{2} d \mathbf{R}},
$$

where $\Psi$ is the actual wave function, which contains $P$ parameters $\left\{\alpha_{i}\right\}$. Suppose that $\Psi=\Psi_{0}$ corresponds to $\boldsymbol{\alpha}=\mathbf{c}$, for some parameter set c. By Taylor-expanding $\Psi$, it can be shown that

$$
\begin{aligned}
E_{u}= & \frac{\int \Psi_{0} \hat{H} \Psi_{0} d \mathbf{R}}{\int \Psi_{0}^{2} d \mathbf{R}}+\frac{\int \Psi_{0} \hat{H} \sum_{i=1}^{P}\left(\frac{\partial \Psi}{\partial \alpha_{i}}\right)_{\alpha=\mathbf{c}}\left(\alpha_{i}-c_{i}\right) d \mathbf{R}}{\int \Psi_{0}^{2} d \mathbf{R}} \\
& -\frac{\int \sum_{i=1}^{P}\left(\frac{\partial \Psi}{\partial \alpha_{i}}\right)_{\alpha=\mathbf{c}}\left(\alpha_{i}-c_{i}\right) \hat{H} \Psi_{0} d \mathbf{R}}{\int \Psi_{0}^{2} d \mathbf{R}}+\mathcal{O}\left[(\alpha-c)^{2}\right] \\
& =\frac{\int \Psi_{0} \hat{H} \Psi_{0} d \mathbf{R}}{\int \Psi_{0}^{2} d \mathbf{R}}+\mathcal{O}\left[(\alpha-c)^{2}\right],
\end{aligned}
$$


where use has been made of the fact that $\hat{H}$ is Hermitian. Hence, $\forall i \in\{1, \ldots, P\}$,

$$
\left(\frac{\partial E_{u}}{\partial \alpha_{i}}\right)_{\boldsymbol{\alpha}=\mathbf{c}}=0
$$

so the wave function used to generate the configuration set corresponds to a stationary point of the unreweighted energy. For a finite number of configurations this result is approximate.

\section{APPENDIX B: PROOF THAT THE UNREWEIGHTED ENERGY HAS A MAXIMUM IN THE SPACE OF LINEAR JASTROW PARAMETERS}

Consider the expression for the quadratic local energy given in Eq. (12). In order to establish that the local energy has a maximum, it must be shown that the eigenvalues of the symmetric matrix $g_{i j}^{(2)}$, which is defined in Eq. (15), are positive. Suppose that

$$
\sum_{j=1}^{P} g_{i j}^{(2)} \alpha_{j}=\lambda \alpha_{i}
$$

where $\alpha_{i}$ is nonzero for at least one $i$. By inserting the definition of $g_{i j}^{(2)}$, multiplying through by $\alpha_{i}$, and summing over $i$, it is found that the eigenvalues $\lambda$ satisfy

$$
\lambda=\frac{\left|\sum_{i=1}^{P} \alpha_{i} \nabla f_{i}\right|^{2}}{\sum_{i=1}^{P} \alpha_{i}^{2}} \geqslant 0 .
$$

(The strict inequality holds provided that $\left\{\nabla f_{i}\right\}$ is linearly independent.) The eigenvalues of $g_{i j}^{(2)}$ are positive and so each quadratic local energy has a maximum in parameter space.

The unreweighted energy is the average of the quadratic local energies of a fixed set of configurations; it is therefore a quadratic function with a maximum in parameter space.

\section{APPENDIX C: CONSTRUCTING THE QUARTIC POLYNOMIAL CORRESPONDING TO A LINE IN PARAMETER SPACE}

Consider the expression for the quartic unreweighted variance as a function of the linear parameters [Eq. (34)], and consider a line in parameter space

$$
\boldsymbol{\alpha}(t)=\mathbf{A}+\mathbf{B} t,
$$

where $\boldsymbol{\alpha}=\left(\alpha_{1}, \ldots, \alpha_{P}\right)$ and $\mathbf{A}$ and $\mathbf{B}$ are constant vectors. The unreweighted variance along the line is given by

$$
\sigma_{u}^{2}(t)=\frac{N_{C}}{N_{C}-1}\left(\Omega_{4} t^{4}+\Omega_{3} t^{3}+\Omega_{2} t^{2}+\Omega_{1} t+\Omega_{0}\right),
$$

where

$$
\Omega_{4}=\sum_{i=1}^{P} B_{i} \sum_{j=i}^{P} B_{j} \sum_{k=j}^{P} B_{k} \sum_{l=k}^{P} B_{l} \Gamma_{i j k l}^{(4)},
$$

$$
\begin{aligned}
\Omega_{3}= & \sum_{i=1}^{P} A_{i} \sum_{j=i}^{P} B_{j} \sum_{k=j}^{P} B_{k} \sum_{l=k}^{P} B_{l} \Gamma_{i j k l}^{(4)} \\
& +\sum_{i=1}^{P} B_{i} \sum_{j=i}^{P} A_{j} \sum_{k=j}^{P} B_{k} \sum_{l=k}^{P} B_{l} \Gamma_{i j k l}^{(4)} \\
& +\sum_{i=1}^{P} B_{i} \sum_{j=i}^{P} B_{j} \sum_{k=j}^{P} A_{k} \sum_{l=k}^{P} B_{l} \Gamma_{i j k l}^{(4)} \\
& +\sum_{i=1}^{P} B_{i} \sum_{j=i}^{P} B_{j} \sum_{k=j}^{P} B_{k}\left(\Gamma_{i j k}^{(3)}+\sum_{l=k}^{P} A_{l} \Gamma_{i j k l}^{(4)}\right),
\end{aligned}
$$

$$
\begin{aligned}
\Omega_{2}= & \sum_{i=1}^{P} A_{i} \sum_{j=i}^{P} A_{j} \sum_{k=j}^{P} B_{k} \sum_{l=k}^{P} B_{l} \Gamma_{i j k l}^{(4)} \\
& +\sum_{i=1}^{P} A_{i} \sum_{j=i}^{P} B_{j} \sum_{k=j}^{P} A_{k} \sum_{l=k}^{P} B_{l} \Gamma_{i j k l}^{(4)} \\
& +\sum_{i=1}^{P} A_{i} \sum_{j=i}^{P} B_{j} \sum_{k=j}^{P} B_{k}\left(\Gamma_{i j k}^{(3)}+\sum_{l=k}^{P} A_{l} \Gamma_{i j k l}^{(4)}\right) \\
& +\sum_{i=1}^{P} B_{i} \sum_{j=i}^{P} A_{j} \sum_{k=j}^{P} A_{k} \sum_{l=k}^{P} B_{l} \Gamma_{i j k l}^{(4)} \\
& +\sum_{i=1}^{P} B_{i} \sum_{j=i}^{P} A_{j} \sum_{k=j}^{P} B_{k}\left(\Gamma_{i j k}^{(3)}+\sum_{l=k}^{P} A_{l} \Gamma_{i j k l}^{(4)}\right) \\
& +\sum_{i=1}^{P} B_{i} \sum_{j=i}^{P} B_{j}\left[\Gamma_{i j}^{(2)}+\sum_{k=j}^{P} A_{k}\left(\Gamma_{i j k}^{(3)}+\sum_{l=k}^{P} A_{l} \Gamma_{i j k l}^{(4)}\right)\right],
\end{aligned}
$$

$$
\begin{aligned}
\Omega_{1}= & \sum_{i=1}^{P} B_{i}\left\{\Gamma_{i}^{(1)}+\sum_{j=i}^{P} A_{j}\left[\Gamma_{i j}^{(2)}+\sum_{k=j}^{P} A_{k}\left(\Gamma_{i j k}^{(3)}+\sum_{l=k}^{P} A_{l} \Gamma_{i j k l}^{(4)}\right)\right]\right\} \\
& +\sum_{i=1}^{P} A_{i} \sum_{j=i}^{P} B_{j}\left[\Gamma_{i j}^{(2)}+\sum_{k=j}^{P} A_{k}\left(\Gamma_{i j k}^{(3)}+\sum_{l=k}^{P} A_{l} \Gamma_{i j k l}^{(4)}\right)\right] \\
& +\sum_{i=1}^{P} A_{i} \sum_{j=i}^{P} A_{j} \sum_{k=j}^{P} B_{k}\left(\Gamma_{i j k}^{(3)}+\sum_{l=k}^{P} A_{l} \Gamma_{i j k l}^{(4)}\right) \\
& +\sum_{i=1}^{P} A_{i} \sum_{j=i}^{P} A_{j} \sum_{k=j}^{P} A_{k} \sum_{l=k}^{P} B_{l} \Gamma_{i j k l}^{(4)},
\end{aligned}
$$

$$
\begin{aligned}
\Omega_{0}= & \Gamma^{(0)}+\sum_{i=1}^{P} A_{i}\left\{\Gamma_{i}^{(1)}+\sum_{j=i}^{P} A_{j}\left[\Gamma_{i j}^{(2)}+\sum_{k=j}^{P} A_{k}\left(\Gamma_{i j k}^{(3)}\right.\right.\right. \\
& \left.\left.\left.+\sum_{l=k}^{P} A_{l} \Gamma_{i j k l}^{(4)}\right)\right]\right\} .
\end{aligned}
$$

All the terms that appear in Eqs. (C3)-(C7) can be evaluated within a single loop over $i, j, k$, and $l$. 


\section{APPENDIX D: WAVE-FUNCTION OPTIMIZATION FOR A MODEL SYSTEM}

Consider a particle of unit mass moving in a onedimensional quartic potential $V(x)=x^{4}$. The Hamiltonian for this system is

$$
\hat{H}=-\frac{1}{2} \frac{\partial^{2}}{\partial x^{2}}+x^{4} .
$$

Let the (unnormalized) trial wave function be

$$
\Psi(x)=\exp \left(-\alpha x^{2}\right),
$$

where $\alpha$ is a positive, optimizable parameter. The energy expectation value is

$$
E=\frac{\int \Psi \hat{H} \Psi d x}{\int \Psi^{2} d x}=\frac{8 \alpha^{3}+3}{16 \alpha^{2}}
$$

which takes its minimum of $E=0.6814202224$ when $\alpha$ $=0.9085602965$. The variance ${ }^{12}$ of the energy is given by

$$
\sigma^{2}=\frac{\int \Psi \hat{H}^{2} \Psi d x}{\int \Psi^{2} d x}-\left(\frac{\int \Psi \hat{H} \Psi d x}{\int \Psi^{2} d x}\right)^{2}=\frac{4 \alpha^{6}-6 \alpha^{3}+3}{8 \alpha^{4}},
$$

which takes its minimum when $\alpha=0.967583472$. The corresponding energy value is $E=0.6840656518$. This illustrates that the variance-minimization method is good, even for an inflexible wave function.

The local energy is given by

$$
E_{L}=\frac{\hat{H} \Psi}{\Psi}=\alpha-2 \alpha^{2} x^{2}+x^{4}
$$

Suppose that a very large number of configurations are distributed according to $\Psi_{0}^{2}$, where $\Psi_{0}=\exp \left(-\beta x^{2}\right)$ for some $\beta$ $>0$. The unreweighted variance of the energy is given by

$$
\sigma_{u}^{2}=\frac{\int \Psi_{0}^{2} E_{L}^{2} d x}{\int \Psi_{0}^{2} d x}-\left(\frac{\int \Psi_{0}^{2} E_{L} d x}{\int \Psi_{0}^{2} d x}\right)^{2}=\frac{4 \beta^{2} \alpha^{4}+3-6 \beta \alpha^{2}}{8 \beta^{4}} .
$$

The value of $\alpha$ that minimizes $\sigma_{u}^{2}$ for a given $\beta$ is

$$
\alpha=\frac{1}{2} \sqrt{\frac{3}{\beta}} .
$$

This is an explicit demonstration of the fact that the parameter values obtained by unreweighted variance minimization depend on the configuration distribution.

As described in Sec. II, when performing variance minimization in practice, one normally repeats the optimization process several times. At each stage the configurations are distributed according to the most-recently optimized wave function. In this simple model, repeated unreweighted variance minimizations generate a sequence of values of $\alpha$, starting with $\alpha_{0}=\beta$ and satisfying $\alpha_{n}=(1 / 2) \sqrt{3 / \alpha_{n-1}}$. The stationary point of this process, irrespective of the initial value of $\beta$, is $\alpha_{\infty}=0.9085602965$. Hence, in this case, selfconsistent unreweighted variance minimization gives the same result as energy minimization, but a different result from that obtained by straightforward variance minimization.

If the trial wave function for this system is

$$
\Psi(x)=\exp \left(-\alpha x^{2}-(x-1)^{2}\right)
$$

then energy minimization, variance minimization, and selfconsistent variance minimization give different results.
${ }^{1}$ W. M. C. Foulkes, L. Mitas, R. J. Needs, and G. Rajagopal, Rev. Mod. Phys. 73, 33 (2001).

${ }^{2}$ C. J. Umrigar, K. G. Wilson, and J. W. Wilkins, Phys. Rev. Lett. 60, 1719 (1988)

${ }^{3}$ P. R. C. Kent, R. J. Needs, and G. Rajagopal, Phys. Rev. B 59, 12344 (1999).

${ }^{4}$ R. J. Needs, M. D. Towler, N. D. Drummond, and P. R. C. Kent, CASINO version 1.7 User Manual (University of Cambridge, Cambridge, England 2003).

${ }^{5}$ N. D. Drummond, M. D. Towler, and R. J. Needs, Phys. Rev. B 70, 235119 (2004).

${ }^{6}$ W. H. Press, S. A. Teukolsky, W. T. Vetterling, and B. P. Flannery, Numerical Recipes in Fortran 77, 2nd ed. (Cambridge University Press, Cambridge, England 1992).
${ }^{7}$ Y. Lee, Ph.D. thesis, University of Cambridge, Cambridge, England 2002.

${ }^{8}$ By a "high-quality" Jastrow factor, we mean a flexible Jastrow factor that can be successfully optimized by variance minimization (i.e., not over-parametrized).

${ }^{9}$ P. Lopez Rios, (private communication).

${ }^{10}$ C. Filippi and C. J. Umrigar, J. Chem. Phys. 105, 213 (1996).

${ }^{11}$ A. J. Williamson, R. Q. Hood, and J. C. Grossman, Phys. Rev. Lett. 87, 246406 (2001).

${ }^{12}$ The variance, which is the quantity estimated in a reweighted QMC variance-minimization calculation, can be calculated exactly for this model system. So we simply refer to "variance minimization" rather than "reweighted variance minimization." 